$\begin{array}{cl}\begin{array}{cl}\text { Revue } \\ \text { de /histoire }\end{array} & \text { Revue de l'histoire des religions } \\ \text { des religions } & 2 \text { | } 2006 \\ & \text { Varia }\end{array}$

\title{
Homéophonies magiques
}

Le rituel en l'honneur de Tacita dans Ovide, Fastes, 2, 569 sq.

Homoeophonic magic. The Ritual for Tacita in Ovid, Fasts, 2, 569 ff

\section{Maurizio Bettini}

\section{(2) OpenEdition}

Journals

Édition électronique

URL : http://journals.openedition.org/rhr/5142

DOI : $10.4000 /$ rhr.5142

ISSN : 2105-2573

Éditeur

Armand Colin

Édition imprimée

Date de publication : 1 juin 2006

Pagination : 149-172

ISBN : 2200-92104-7

ISSN : 0035-1423

Référence électronique

Maurizio Bettini, «Homéophonies magiques », Revue de l'histoire des religions [En ligne], 2 | 2006, mis en ligne le 20 janvier 2010, consulté le 21 avril 2019. URL : http://journals.openedition.org/rhr/5142 ; DOI : 10.4000/rhr.5142 


\section{Homéophonies magiques Le rituel en l'honneur de Tacita dans Ovide, Fastes, 2, 569 sq.}

Dans les Fastes (2, 569 sq), Ovide décrit un rituel pour la déesse Tacita qui a le but de bloquer les médisances. Si dans quelques passages, les associations symboliques utilisées apparaissent banales, dans d'autres, on est devant des rapports inédits et plutôt déconcertants. Par exemple: pourquoi la vieille célébrante, afin de "bloquer les langues hostiles», introduit "trois grains d'encens » dans le trou qu'une souris a creusé sous le seuil? Et pourquoi mâche-t-elle sept fèves noires? À notre avis, la réponse à ces questions se trouve dans le genre de magie ici décrit par Ovide : non seulement " homéopathique », mais aussi " homéophonique ", c'est-à-dire accomplie par associations symboliques de caractère phonique et verbal.

\section{Homoeophonic magic. The Ritual for Tacita in Ovid, Fasts, 2, 569 ff.}

In Fasti (2, 569 ff.), Ovid describes a ritual for the goddess Tacita, the purpose of which is to ward off gossip. If in some passages the symbolic associations appear predictable, elsewhere we are faced with relationships that are unexpected and at times quite disconcerting. For example: why, in order to "obstruct unfriendly tongues", does the celebrant - an old woman - put "three grains of incense" in the opening that a mouse has made beneath the threshold? And why does she chew on "seven black fava beans"? In my opinion, the answer to these questions rests in the type of magic that Ovid describes here: not only "homeopathic", but also "homeophonic" — that is, realized through symbolic associations of a phonic and verbal nature. 
À mon ami Alessandro Barchiesi, magister ludi.

Dans sa Vie de Numa, Plutarque raconte ce qui suit : « [Numa] prescrivit aux Romains de vénérer tout particulièrement avec insistance une Muse, celle à qui il donna le nom de Tacita (Toxítav $\pi \rho 0 \sigma \alpha \gamma o \rho \varepsilon v ́ \sigma \alpha \varsigma)$, autrement dit la "silencieuse" $(\sigma \iota \omega \pi \eta \lambda \eta)$ ou la "muette" ( $\dot{\varepsilon} \vee \varepsilon \alpha)$, chose qui semble être propre à qui rappelle et honore la réserve $(\dot{\varepsilon} \chi \varepsilon \mu v \theta i ́ \alpha)$ pythagoricienne. ${ }^{1} \gg$ Numa indique donc expressément aux Romains de vénérer au plus haut point une "Muse" muette et silencieuse, une muse de la réserve, portant un nom, pour une déesse muette, en vérité, bien "parlant": Tacita ${ }^{2}$. Nom dont la morphologie d'abord se révèle significative, s'agissant d'un substantif en - $t a$, réalisé sur le verbe tace-o « se taire » (Taceta $>$ Taci-ta). En général, ce type de substantif déverbal implique une notion d'activité, donnant l'impression de véritables nomina agentis. Il suffit de penser à Moneta «qui fait rappeler», Genita « qui fait naître », Stata mater «la déesse qui arrête l'incendie », etc. Ainsi Tacita serait-elle à proprement parler « la déesse qui fait taire $^{3} »$. Mais que savons-nous du rituel qui lui était consacré ?

1. Plutarque, Vita Numae, 8 ; $c f$. Jean Gagé, Apollon Romain, E. de Boccard, Paris, 1955, p. 337.

2. Il est très probable que Tacita ait fait partie du groupe des Camenae : voir Plutarque, op. cit., 13 ; Georg Wissowa, Religion und Kultus der Römer, Beck, München, 1971 (=1972), p. 219, 235 ; Gerard Radke, Die Götter Altitaliens, Münster, Aschendorff, 1965, s. v. Camenae, p. 78 sq. ; sur Tacita, voir Françoise Bader, «Langue liée et bouche cousue. Ovide, Fastes, 2, 571-582 », Revue de philologie, $\mathrm{n}^{\circ}$ 66, 1992, p. 217-245 ; et surtout Anne Dubourdieu, « Divinités de la parole dans la Roma antique », Revue de l'histoire des religions, 220, 3/2003, p. 259-282, qui étend son enquête à une comparaison avec Angerona et Aius Locutius.

3. Cf. Albrecht von Blumenthal, «Zur Römischer Religion des archaischen Zeit », II, Reinisches Museum 90, 1941, p. 310-334. Etant donné que les substantifs en -ta peuvent aussi exprimer généralement la notion contenue dans le verbe, on ne peut exclure que Tacita ait été simplement la « déesse qui représente le silence » : $c f$. Radke, op. cit. s. v. Tacita, p. 295 sq., qui suggère la possibilité d'interpréter Tacita aussi comme «göttliche Abstraktion » du silence du culte ; Franz Bömer, P. Ovidius Naso. Die Fasten. II, Carl Winter, Heidelberg, 1958, p. 30 sq., 127 ; Dubourdieu, op. cit. 


\section{Un RITUEL POUR TACITA}

Tous les 21 février, Rome fêtait les Feralia, le jour des morts, seul jour des dies parentales appartenant aussi aux feriae publicae. Les dies parentales commençaient le 13 février, inaugurant une période où les autorités déposaient la toga praetexta, les temples restaient fermés, les mariages étaient interdits, laissant à chacun le loisir d'offrir des sacrifices pour honorer les âmes des défunts. Ovide nous décrit dans ses Fastes le curieux rituel qui se serait déroulé à l'occasion des Feralia ${ }^{4}$ :

«Cette date porte le nom de Feralia parce qu'on porte (ferre) aux morts les offrandes qui leur sont dues : c'est le dernier jour pour apaiser les Mânes.

Voici une vieille décrépite qui, assise parmi les jeunes filles, célèbre un sacrifice à Tacita - mais elle, elle a du mal à se taire. Avec trois doigts, elle pose trois grains d'encens sous le seuil, à l'endroit où une souris s'est ouvert un passage. Puis elle attache avec du sombre plomb sept fils sur lesquels elle a prononcé un enchantement, elle mâchonne en bouche sept fèves noires. Alors elle prend une tête d'anchois et - après l'avoir passée dans de la poix et cousue avec une aiguille de bronze elle la brûle dans le feu, en versant dessus du vin. Ce qui reste, elle le boit avec ses compagnes - c'est elle en tout cas qui en boit le plus. "Nous avons attaché les langues hostiles et les bouches ennemies", ainsi dit la

4. Ovide, Fastorum libri, 2, 569 sq.

Hanc, quia iusta ferunt, dixere Feralia lucem;

ultima placandis manibus illa dies.

Ecce anus in mediis residens annosa puellis sacra facit Tacitae (vix tamen ipsa tacet), et digitis tria tura tribus sub limine ponit, qua brevis occultum mus sibi fecit iter: tum cantata ligat cum fusco licia plumbo, et septem nigras versat in ore fabas, quodque pice adstrinxit, quod acu traiecit aena, obsutum maenae torret in igne caput ; vina quoque instillat: vini quodcumque relictum est, aut ipsa aut comites, plus tamen ipsa, bibit.

"Hostiles linguas inimicaque vinximus ora» dicit discedens ebriaque exit anus.

Protinus a nobis quae sit dea muta requires: disce per antiquos quae mihi nota senes.

Sur muta (v. 583) sans majuscule, $c f$. infra. 
vieille en s'éloignant, et elle sort saoule. Maintenant, tu me demanderas qui est cette déesse muette. Alors écoute ce que j'ai appris des plus vieux (...). »

Tacita, muse chère à Numa, était donc honorée à Rome au cours des Feralia. De plus, le rituel décrit par Ovide confirme aussi bien les indications qu' on peut tirer du nom de la divinité (Taci-ta « celle qui fait taire »), que la brève description de Plutarque. Les sacra célébrés en l'honneur de Tacita ont en effet pour but déclaré de «faire taire » les mauvaises langues ( Nous avons attaché les langues hostiles et les bouches ennemies » $)^{5}$. Tacita est donc invoquée ici en tant que déesse qui protège du mal provoqué par la médisance des autres, autrement dit de leur hostilité verbale. La sphère où se meut notre Muse du silence commence à s'éclairer : on peut s'adresser à elle pour se défendre de ses propres excès de loquacité, comme le recommandait Numa, mais aussi pour se protéger de ceux des autres. Tout en rendant ses adeptes plus réservés, Tacita les met aussi bien à l'abri de l'indiscrétion et de la médisance des autres. On sait qu'à Rome, entrer dans le discours des autres - en tant qu' objet - créait plus d'une crainte ${ }^{6}$.

\section{Historiola}

Chez Ovide, la description un peu bizarre de ce rituel est suivie d'un récit mythologique, qui avait pour fonction d'expliquer quae sit dea muta « qui est cette déesse muette », autrement dit la mystérieuse Tacita honorée par la vieille. Avant de passer à l'analyse du rituel, il n'est pas indifférent de résumer brièvement ce récit ${ }^{7}$.

5. Cette intention est très récurrente dans les tabellae defixionum. Cf. Auguste Audollent, Defixionum tabellae, Fontemoing, Paris, 1904 : 25, 16 ; 27, 10 ; 66 ; 112, 6 ; 139 ; 192, 6 ; 221 ; 222 ; etc. ; Bader, op. cit., p. 227 ; Dubourdieu, op. cit., p. 276 n. 70 ; Frédéric Chapot et Bernard Laurot, Corpus de prières grecques et romaines, Brepols, 2001, p. 349-351, n. L78, note.

6. J'ai traité ce thème dans Le orecchie di Hermès, Einaudi, Torino, 2000, p. 20-33.

7. vv. 583-616. 
Dans le Latium vivait une naïade répondant au nom de Lara, qui en fait se prononçait d'abord Lala ( $\lambda \alpha \alpha^{\lambda} \lambda$ en grec signifie « bavarde »); c'est seulement par la suite qu'il se transforma en Lara. Cette jeune fille était terriblement loquace. Son père, le fleuve Almon, l'avait avertie plus d'une fois : Nata, tene linguam! «Ma fille, tiens ta langue $!^{8} \gg$ Mais elle n'écoutait pas les conseils de son père. Un jour, Jupiter tomba amoureux de la nymphe Juturne, qui se mit à le fuir. Le père des hommes et des dieux convoqua les nymphes, et les informa de son intention de profiter de leur aide pour satisfaire ses désirs. Lara n'eut qu'une hâte, celle de révéler à Juturne qu'il avait l'intention de la prendre par la force et à Junon que son mari la trahissait avec Juturne. L'ire de Jupiter fut impitoyable, il infligea à Lala/Lara une horrible punition : " Puisqu'elle n'avait pas su en faire bon usage, il lui arrache la langue. ${ }^{9}$ » Sur ce, il appela Mercure et lui ordonna d'accompagner la coupable dans les marécages de l'enfer, dont elle serait désormais la nymphe : ce lieu «silencieux» conviendrait à Lara, désormais muette. Mais au cours du voyage, Mercure, éprouvant une certaine attraction pour la jeune fille, la viola. Les suites de cette violence firent concevoir à Lara deux jumeaux, les Lares (autre jeu de mots facile, cette fois entre Lara et Lares), ceux qui aujourd'hui encore veillent aux compita, autrement dit aux carrefours.

Telle est l'historiola mythique - s'il est permis de la définir comme telle ${ }^{10}$ - qui, selon Ovide, expliquerait au lecteur « qui est cette déesse muette ». Observons-en de plus près le noyau central. Il tourne autour d'une affaire d'indiscrétion, autrement dit du fait de parler trop. Lala/Lara, la naïade qui parle à tort et à travers, ne

8. v. 602 .

9. v. 697 sq.

10. Dans la tradition des études sur le folklore antique et moderne, ce terme est plutôt réservé à la « mythistoire » qui précède ou qui suit une conjuration magique, pour en fonder l'efficacité. Ce «genre littéraire » avait déjà été identifié comme tel par les pères fondateurs de la discipline comme Grimm, Kuhn, Dietrich, etc., (voir R. Heim, «Incantamenta magica Graeca et Latina », Jahrbücher für Klassiche Philologie 19, Supplementband, 1893, p. 495 sq.) ; c'est dans ce sens que l'utilisera encore Ernesto De Martino, Sud e magia, Feltrinelli, Milano, 1959. Henri Le Bonniec, Les Fastes, Catania, Orpheus, 1969, I, 128, définit le récit ovidien comme une « historiette ». 
mérite pas sa langue, on la rend muette. L'identité d'origine de la déesse Tacita est donc celle d'une divinité qui assume son statut per antiphrasin: on passe d'un excès de mots (Lala/Lara, la nymphe bavarde) à un défaut de parole (Tacita, la déesse muette). Toute l'histoire, telle que le poète la présente, est animée d'une vigoureuse misogynie, véhiculant une idée de fond, à savoir que les femmes ne se taisent jamais. On se souvient qu' au cours du rituel, Ovide a soin de préciser que, bien qu'elle sacrifie à une déesse appelée Tacita, la vieille vix tamen ipsa tacet «a vraiment du mal à se taire ${ }^{11}$ ». Bref, une vieille qui offre un sacrifice à Tacita en baragouinant, en « mâchonnant », une nymphe appelée « bavarde » qui vend la mèche et trahit les secrets des hommes, la morale de cet épisode semble être la suivante : les femmes ne peuvent se taire que si on leur arrache la langue. Dans ce cas, la divinité semble représenter une hypostase de la punition du caractère loquace des femmes.

Considérons la partie finale de cette historiola, la condamnation de Lara et la violence que lui inflige Mercure. Ces deux dernières séquences fonctionnent comme une sorte de relais, l'explication narrative de l'identité de Tacita s'y relie au calendrier et au contexte religieux des Fastes en général. Le bref épisode où Jupiter condamne Lara à devenir une nymphe des marais de l'enfer (et en tant que telle, silencieuse) produit, en effet, sa motivation : cet épisode nous explique pourquoi une nymphe, impliquée dans des affaires d'amours divines, a pu devenir Tacita, la déesse célébrée le jour funèbre des Feralia. Seul un écrivain doté d'imagination narrative tel qu'Ovide pouvait inventer une corrélation entre le silence de Lara « réduite au mutisme » et le silence des morts : cette association entre le fait d'être « réduite au silence » et le marais silencieux des morts fait de la nymphe Lara un avatar crédible de la déesse muette Tacita honorée lors des Feralia. Quant à l'épisode final de l'historiola, retraçant le voyage de Mercure, la violence subie par la nymphe et la naissance des jumeaux, il sert à créer une généalogie aux Lares, divinités dont nous connaissons l'importance dans la culture romaine. Loin d'être original, cet épisode suit un modèle fréquent dans la mythologie

11. v. 572. 
grecque et romaine : une divinité exerce sa violence ou sa séduction sur une mortelle, déterminant une naissance, ici celle des demi-dieux jumeaux ${ }^{12}$. On aurait tort de sous-évaluer ces séquences de relais qui concluent le récit, bien qu'elles établissent des relations entre des histoires et des personnages qui, vraisemblablement, n'existaient pas avant Ovide, ou avaient peu de rapport entre eux ${ }^{13}$. De telles inventions narratives contribuent précisément à la naissance progressive d'une «mythologie romaine », projet auquel l'auteur des Fastes travaillait.

\section{MÉdisAnCE ET JEUX DE MOTS}

Quel est l'élément qui nous frappe le plus dans cette historiola imaginée par Ovide ? Probablement la force de la composante ludique de son invention littéraire : sans l'ironie misogyne du poète, sans ses jeux de mots, sans les rapports plutôt libres et arbitraires qu'il établit entre les personnages, cet épisode n'existerait pas. Ce qui frappe en particulier, c'est l'intérêt que manifeste Ovide pour les calembours. Tout l'épisode, en effet, est construit sur le rapport étymologique établi entre $\lambda \alpha^{\prime} \lambda \eta$ ( «bavarde») et «Lara ». Cette curieuse origine grecque qu'Ovide attribue au nom Lara, bien enraciné en fait dans la

12. Le thème de Hermès violeur de jeunes filles est récurrent dans la mythologie grecque : $c f$. Laurent Gourmelen, Kékrops, le Roi Serpent, les Belles Lettres, Paris, 2005, p. 163 sq.

13. Les savants s'accordent pour qualifier cette petite histoire de «Erfindung » ovidienne (Wissowa, Religion und Kultus der Römer, op. cit., p. 235 ; Radke, op. cit., p. 225 ; Henri Le Bonniec, Ovide, Fastorum Liber II, PUF, Paris, 1969 ; etc.). Il faut cependant préciser que, même si Ovide a manifestement «inventé » une bonne partie de ce récit - mais tout récit mythologique n'est-il pas une invention ? Existe-t-il des versions «fausses » et des versions « vraies » en mythologie ? -, je ne crois pas qu'il ait inventé une dea Muta, comme semblait le penser Wissowa (Römische Sagen, dans Gesammelte Abhandlungen zur römischen Religions- und Stadtgeschichte, Beck, München, 1904 [= New York, Arno Press, 1975], p. 140 sq.), et comme d'autres l'ont soutenu après lui, ce qui a donné lieu à une encore moins crédible Tacita Muta. Cf. Le Bonniec, Ovide, Fastorum liber II, op. cit., et surtout Dubourdieu, op. cit. On pourrait soutenir l'existence de divinités Mutae Tacitae, au pluriel, sur la base de la tablette magique (n. L78, Chapot, Laurot, op. cit.) dont nous nous occuperons, $c f$. infra. 
tradition romaine ${ }^{14}$, lui permet de définir le caractère de son personnage en le tirant de l'étymologie de son nom. La fin de l'histoire repose sur un jeu parétymologique analogue, entre «Lara» et «Lares». N'oublions pas cette propension d'Ovide pour les calembours : dans la deuxième partie de notre analyse, elle va nous permettre d'interpréter quelques-unes des opérations magiques accomplies par la vieille femme. Pour le moment, revenons à la scène décrite par Ovide.

On l'a vu, ce rituel représente Tacita comme une déesse qui « fait taire » la médisance des autres, bloquant leur hostilité verbale. La vieille femme le déclare explicitement à la fin du rite, quand elle prononce la phrase : Hostiles linguas inimicaque vinximus ora, « Nous avons attaché les langues hostiles et les bouches ennemies ${ }^{15}$. Bref, elle est convaincue d'avoir bloqué, au moyen des sacra consacrés à la déesse, ce que les Latins appelaient obtrectationes ou maledicta, mais aussi rumores, rumusculi, fabulae, murmura, murmurationes et ainsi de suite. Il s'agit donc d'une divinité - provient-elle de l'univers des antiques «divinités fonctionnelles » romaines ${ }^{16}$ ? qui gouvernait la province du discours corrosif, à savoir de la perfide médisance. Comment procède la vieille femme pour arriver aux fins qu'elle se propose ? Au moyen d'actions qui ont un caractère nettement magique. Il s'agit d'une série de tours de magie assez curieux, dont les passages offrent quelque difficulté de compréhension ${ }^{17}$. Voyons dans l'ordre chaque étape de ce rituel :

14. $C f$. des noms comme Larenta, Larentia, Larunda.

15. v. 581 .

16. Micol Perfigli, Indigitamenta. Divinità funzionali e Funzionalità divina nella Religione Romana, ETS, Pisa, 2004.

17. James George Frazer, Publii Ovidi Nasonis Fastorum libri sex, II, Macmillan, London, 1929, p. 446 : «Some of the details of the magic rite performed by the ancient dame are obscure »; Bömer (op. cit., II, 126) invite à ne pas vouloir "interpréter de façon trop précise » ce rituel de la vieille femme ; voir aussi Anne-Marie Tupet, La magie dans la poésie latine, les Belles Lettres, Paris, 1976, p. 408 sq. À titre de curiosité, on peut citer à ce propos l'interprétation de Carolus Neapolis, Anaptyxis ad Fastos Ovidianos, Antuerpiae, 1639 (cité par Thomas Keightley, dans Ovid's Fasti, Whittaker, London, 1839, p. 65) selon laquelle, le lien entre le rituel célébré par la vieille femme, destiné à bloquer les médisances, et la fête des morts, avait pour fin de mettre en pratique la maxime de mortuis nihil nisi bonum. 
- la vieille femme place, avec trois doigts, trois grains d'encens sous la porte, à l'endroit où une souris a creusé son passage ;

- puis elle attache avec du plomb des fils sur lesquels elle a pratiqué un enchantement ;

- elle mâchonne (versat) en bouche sept fèves noires ;

- elle prend une tête de maena, autrement dit d' " anchois » (en grec $\mu \alpha \tilde{\imath} \nu \alpha, \mu \alpha \iota v i(s)$, elle l'emprisonne dans une couche de poix, la coud avec une aiguille de bronze, puis la brûle, elle l'arrose de vin et boit ce qui reste du vin ;

- enfin elle déclare, on l'a vu, qu'elle a "attaché les langues hostiles et les bouches ennemies ».

Essayons à présent de désarticuler ce rituel, en analysant chaque opération. Commençons par celles qui nous paraissent plus claires.

\section{FILS DE PLOMB, POIX ET TÊTES D’ANCHOIS}

Bon nombre d'enchantements consistent dans le fait de nouer des fils. Frazer et Bömer ${ }^{18}$ fournissent de nombreux parallèles de cette procédure magique. Le plus connu de ces parallèles se trouve dans la huitième Bucolique de Virgile, où une femme abandonnée cherche à récupérer celui qu'elle aime par la magie : « de ces fils trois par trois je t'enroule, différents par leurs trois couleurs ${ }^{19}$ ». Mais, sans recourir à de tels parallèles, il est clair que l'action de ligare, de produire des nœuds, implique toujours dans la magie l'intention de « bloquer ${ }^{20}$ », vincire, qui est précisément le but de

18. Frazer, op. cit., II, p. 448 sq. ; Bömer, op. cit., II, 126 ; d'autres parallèles dans Samson Eitrem, Classical Revue 36, 1922, p. 72 ; Le Bonniec, Ovide, Fastorum liber II, op. cit. ; voir aussi la belle analyse de Dubourdieu à propos de Angerona, la déesse qui se présente ore obligato obsignatoque, autrement dit praenexo obisgnatoque ore (Masurius Sabinus, dans Pline, Naturalis historia 3, 65 et Macrobe, Saturnalia 1, 10, 7 ; Solinus, Collectanea, I, 6).

19. Virgile, Bucoliques, 8, $73 \mathrm{sq}$ : terna tibi haec primum triplici diversa colore / licia circumdo.

20. Voir mon étude Nascere. Storie di donne, donnole, madri e eroi, Einaudi, Torino, 1998, p. 92-96. 
ce rituel. Procédure magique que Frazer aurait appelée «Homoeopathic $^{21} »$ : en liant (ligat) les fils avec du plomb, la vieille femme entend lier (vinximus) les langues et les bouches des médisants et des faiseurs de ragots. Quant au choix spécifique du plomb pour accomplir cette opération, les Romains, comme les Modernes, utilisaient ce métal en fusion pour souder divers objets, pour plumbare ${ }^{22}$. Dans ce sens, ce matériel était étroitement associé à l'image du «blocage »: le processus magique pouvait facilement tirer profit de cette association.

Considérons la tête de maena, ou anchois, sur laquelle l'exécutante semble s'acharner tout particulièrement. Ici aussi, l'intention de «bloquer» paraît évidente : en effet, avant de la brûler, elle enferme le maenae...caput dans une couche de poix et la coud avec une aiguille en bronze ${ }^{23}$. La fonction symbolique de la tête de maena est évidente, elle représente les linguae/ora qu'il faut ligare, nous avons affaire à un autre procédé de magie homéopathique. Mais pourquoi précisément une tête de maena?

Certes, nul n'ignore que les poissons sont « muets ». Des manipulations qui veulent produire le mutisme requièrent un être vivant déjà pourvu de cette qualité ${ }^{24}$. Mais une petite histoire, que nous aborderons aussi plus loin, nous garantit que, dans la tradition romaine, l'anchois (maena) assumait précisément la fonction de substitut symbolique de la personne humaine. Il s'agit de l'épisode mythique où Numa demande à Jupiter de lui enseigner les règles pour l'expiation de la foudre. Le père des hommes et des dieux demande trois fois au roi d'accomplir en échange un sacrifice humain - et par trois fois, Numa use d'un astucieux jeu de mots

21. Dans la mesure où elle est basée sur « the Law of Similarity » : James George Frazer, The Golden Bough, I, The Magic Art and the Evolution of Kings, Macmillan, London, 1911, p. 53 sq. ; il renvoyait pour l'expression « Homeoepathic Magics » à Yrjo Hirn, Origins of Art, London, 1900.

22. Cf. par ex. Caton, De agricultura, 20 et 21; Pline, Naturalis historia, 34,161 ; etc.

23. Sur le fait que l'aiguille était en bronze, $c f$. la note de Frazer, Publii Ovidi Nasonis Fastorum libri sex, op. cit., II, p. 451 sq. ; on ne peut franchement pas démontrer que l'instrument utilisé par la vieille femme était une sabula, « alène », et non une aiguille, comme l'a soutenu Bader.

24. Cf. Bömer, op. cit., II, 127. 
pour « détourner» les requêtes de Jupiter vers des objets absolument inoffensifs : respectivement un « oignon », des « cheveux », et des «anchois $»^{25}$. Le caractère folklorique et populaire de cette petite histoire est hors de doute ${ }^{26}$. Nous pouvons donc affirmer que, dans la tradition symbolique romaine, « anchois » était un bon équivalent de «personne humaine ». Par conséquent, en enfermant cette « tête d'anchois » (maenae...caput), dans de la poix et en la cousant, c'est une tête humaine qu'entendait coudre et enfermer dans de la poix celle qui célébrait les sacra de Tacita : elle versait la poix autour des langues (humaines) médisantes et cousait les bouches (humaines) des potineurs. Et en brûlant finalement cette tête d'anchois, elle met fin à l'existence de ces langues/bouches malveillantes, puis elle les sacrifie d'une aspersion de vin. Il s'agit toujours d'une magie homéopathique.

Jusqu'ici, les choses se présentent de façon assez claire. Mais que dire des sept fèves noires que la vieille «mâchonne dans sa bouche » (versat in ore) ? Il est difficile de percevoir un lien entre cette action et le but - explicité à la fin de la séquence - de bloquer la médisance des autres. Même les commentateurs semblent ici embarrassés. Frazer rappelle le caractère funèbre des fèves, qui sont utilisées aussi à l'occasion des Lemuria $^{27}$ - et l'on sait qu'elles

25. Valerius Antias, fr. 8 Beck, Walter $=$ Arnobe, Adversus nationes 5, 1 (Die frühen Römischen Historiker, II, hrsg. von Hans Beck und Uwe Walter, Wissenschaftliche Buchgesellschaft, Darmstadt, 2005, p. 176 sq.) ; Ovide, Fastorum libri, 3, 329 sq. ; Plutarque, Vita Numae 15, 4 sq. Le texte d'Ovide parle simplement d'un piscis, sans autre détermination ; maena se trouve dans Valerius Antias/Arnobe ( $c f$. infra, n. 41), le texte de Plutarque a roıvíc. Pour l'éventualité d'un rapport de cette histoire avec la fête des Volcanalia, où l'on brûlait également un piscis, $c f$. Bömer, op. cit., II, 126 (qui le nie).

26. En effet, Macrobe rapporte dans ses Saturnalia, 1, 7, 34 sq., une histoire sur le même schéma de substitution par similarité ; il la réfère cependant à Brutus majeur et l'attribue à la définition des Compitalia : Apollon avait demandé qu'on célèbre au cours de la fête des sacrifices d'êtres humains, désignés par l'expression capita ; mais Brutus avait détourné l'infausta significatio de ce terme en substituant aux humains des capita allii et papaveris ; on retrouve ce schéma de substitution par similarité dans le rituel des Compitalia tel qu'il est décrit dans Festus, 273 L. (aux capita des esclaves correspondent cette fois des pilae).

27. Frazer, Publii Ovidi Nasonis Fastorum libri sex, op. cit., II, 450 sq. ; cf. Ovide, Fastorum libri, 5, 436 sq. ; de même, Le Bonniec, Ovide, Fastorum liber II, op. cit. 
sont liées dans l'Antiquité au monde des morts ; Bömer se borne à remarquer qu' « avec sept fèves noires, Pythagore et l'enchantement vont de pair » et que le «noir» des fèves accentuerait le caractère «noir» de la « magie » de ce rituel ${ }^{28}$. Admettons que les fèves aient une signification funèbre, adéquate aux fêtes des Feralia, et que Pythagore y soit mêlé, mais pour quel motif cette vieille devrait-elle les « mâchonner en bouche » ? Et, de surcroît, sans aucune intention de calmer ou de chasser les ombres des morts, comme le voudrait la signification funèbre des fèves, mais pour bloquer les médisants?

L'autre phase du rituel, que nous n'avons pas encore examinée, se prête à un discours analogue. "Trois grains d'encens » posés «avec trois doigts » à l'endroit où une «souris s'est ouvert un passage sous le seuil », cette opération présente elle aussi une énigme, qui embarrasse les commentateurs. Bömer se tait, Frazer fait de nouveau appel au monde des morts : il s'agirait de grains d'encens offerts aux esprits qui habitent sous le seuil ${ }^{29}$. Soit. Mais que dire de la souris, et de l'ouverture, du «passage » qu'elle se creuse ? Et pourquoi offrir aux morts des grains d'encens, qui plus est en les enfilant dans l'ouverture creusée par une souris, alors qu'on a l'intention d'attacher les langues hostiles et de coudre les bouches ennemies ? Ne disposant pas de parallèles romains, Frazer se voit contraint cette fois à vaquer entre le Maroc, la Palestine, l'Allemagne, le Nyasaland, la Russie et ainsi de suite. Mais ici, comparaison n'est pas raison, car elle ne résout pas nos problèmes.

Pour interpréter ces deux énigmatiques séquences rituelles, nous pourrions imaginer qu'Ovide aurait opéré ici une sorte de saut, une fracture dans le mode de signification du procédé magique. Il garde le même cadre de référence, à savoir la magie analogique, à ceci près : les deux premières opérations analysées se fondent sur des analogies entre des objets et des actions - «lier des fils avec du plomb $»=$ «bloquer le processus »; «enfermer une tête d'anchois

28. Bömer, op. cit., II, 126.

29. Frazer, Publii Ovidi Nasonis Fastorum libri sex, op. cit., II, 447 ; selon Hermann Peter, P. Ovidi Nasonis Fastorum libri sex, Teubner, Leipzig, 1874 , p. 102, la mention de la «souris » serait due au fait que ces animaux avaient une signification prophétique et étaient sacrés à Apollon (?). 
dans de la poix et la coudre »= « engluer des langues humaines et coudre des bouches humaines »-, les analogies à la base de ces deux autres procédures s'établiraient plutôt entre des mots et des sons. En d'autres termes, le rapport d'analogie existant entre les actes de «mâchonner en bouche sept fèves » et de «placer trois grains d'encens sous le seuil, à l'endroit où une souris s'est ouvert un passage », pourrait résider dans des jeux de mots. Mais avant de poursuivre notre analyse, il faut nous assurer de l'éventualité d'un fonctionnement des procédés magiques de type analogique à un niveau purement linguistique. En d'autres termes, voir si dans la culture antique, ce que Frazer appelait «the Law of Similarity » agissait non seulement entre des objets et des actions que l'on jugeait similaires, mais aussi entre des mots et des sons similaires : s'il existait à côté d'une magie «homéopathique», une magie spécifiquement « homéophonique ».

\section{MAGIES HOMÉOPHONIQUES}

Dans son Apologie, Apulée énonce explicitement le modèle de magie en question ${ }^{30}:$ "Quoi de plus stupide que de présupposer que, à partir de la proximité des mots, agisse une force similaire entre les choses? » Accusé de magie, Apulée se défend en taxant de «stupidité » un procédé magique par ailleurs très commun ${ }^{31}$. Pour ne citer que quelques exemples, rappelons le cas de l'herbe reseda, qu'on invoquait, dans un enchantement rapporté par Pline, parce qu'elle avait le pouvoir de morbos resedare; ou du précepte selon lequel uva uvam emendat « le raisin fait du bien à la luette»,

30. Apulée, Apologia, 34: An quicquam stultius quam ex nominum propinquitate vim similem rerum coniectam?

31. $C f$. Adam Abt, «Die Apologie des Apuleius von Madaura und die antike Zauberei », Religiongeschichtliche Versuche und Vorarbeiten, 4, 2, Töpelmann, Giessen, 1908, p. 213 sq. (« Diese Verteidigung des Apuleius ist nicht besonders glücklich. Der Schluss vom Namen auf die Kraft ist in Zauber gern gemacht worden [...]»); Grazia Maria Manselli, «Apuleio mago e l'incanto della parola» in Annali della Facoltà di Lettere e Filosofia, 46, Università degli Studi di Bari, 2003, p. 3-39. 
est efficace contre les maux de gorge ( $u v a$ signifie à la fois « raisin » et « luette $)^{32}$. Cette magie joue de façon « homéopathique » sur la propinquitas entre les nomina pour obtenir une similis vis entre les res : homéophonie magique. Varron nous transmet un enchantement contre les «maux de pieds » qui n'est pas bien différent ${ }^{33}:$ « Tu m'es venu à l'esprit,/soigne mes pieds !/Que la terre garde le mal/ que la santé reste ici/dans mes pieds. » Dans ce texte, la définition de pestem, attribuée au «mal » qui afflige les extrémités de la personne, est clairement «pilotée » par son homéophonie avec pedes.

En dehors de la tradition folklorique, des exemples de ce genre se trouvent dans le domaine de la «magie littéraire », genre assez répandu dans la production antique ${ }^{34}$. Prenons le texte des Magiciennes de Théocrite : Simaitha, abandonnée par Delphis, brûle du laurier pour obtenir un effet analogue sur l'aimé qui l'a trahi ${ }^{35}$ : « Delphis m'a brûlée, et je brûle pour Delphis ce laurier. » Ce qui signifie que le laurier, en brûlant, devra faire brûler (d'amour) Delphis, comme Delphis a brûlé (d'amour trahi) le cœur de Simaitha. Du point de vue des procédures magiques, le mécanisme est clair : il part du choix d'un « matériau » déterminé (le « laurier») ${ }^{36}$ comme l'équivalent de l'objet auquel l'enchantement s'adresse (Delphis); après avoir établi ce rapport, l'action s'accomplit sur cet équivalent, et est censée provoquer ses effets sur l'objet de l'enchantement. Mais le texte grec montre clairement l'importance de l'homéophonie dans le procédé de magie. En effet, Simaitha déclare ${ }^{37}$ : «Delphis

32. Pline, Naturalis Historia, 27, 131 ; Marcellus Empiricus, De medicamentis, 14, 26 ; d'autres exemples dans Abt, op. cit.

33. Varron, De re rustica, 1, 2, 27 : Ego tui memini/medere meis pedibus./ Terra pestem teneto,/salus hic maneto/in meis pedibus ; $c f$. Pline, Naturalis Historia, 28, 21 : fr. 5 Morel.

34. Cf. Tupet, op. cit.; les textes les plus significatifs de ce «genre» singulier sont réunis dans l'anthologie de Georg Luck, Arcana Mundi, I, Mondadori, Milano, 1997, p. 28 sq.

35. Théocrite, Idyllia, 2, 23 sq.

36. Dans la terminologie magique, ce « matériel » choisi aurait probablement le nom de oủoí $\alpha$ «substance» : cf. Papyri Graecae Magicae, 4, 297 sq. (= Luck, op. cit., 16, p. 156) et 4, 2943 sq. (= Luck, op. cit., 24, p. 176).

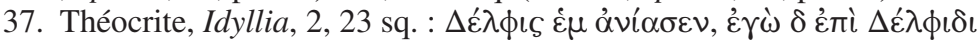
$\delta \alpha \dot{\phi} v \alpha \nu / \alpha i \theta \omega$. 
m'a brûlée, et je brûle pour Delphis ce laurier. » Dans leur contiguïté,

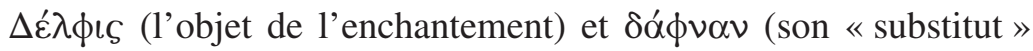
magique) présentent un étroit rapport d'analogie phonique. Si le laurier fait brûler Delphis, c'est parce que le nom du matériau magique rappelle le nom du destinataire de l'enchantement. Simaitha applique le même procédé à une autre phase du rituel. Cette fois, il s'agit de répandre de la farine $(\dot{\alpha} \lambda \phi \iota \tau \alpha)$ sur le feu, elle dit en même temps ${ }^{38}$ : « J'éparpille les os (ỏotí $\alpha$ ) de Delphis. » De nouveau, l'enchanteresse a établi qu'un certain matériau magique (de la «farine») fonctionne comme un équivalent de l'objet (les « os de Delphis») auquel est destiné l'enchantement. Par conséquent, « éparpiller de la farine » signifiera «éparpiller les os de Delphis ». Mais, ici aussi, l'analogie magique est renforcée par l'analogie phonique $(\Delta \hat{\varepsilon} \lambda \phi \iota \delta o \varsigma / \alpha \dot{\alpha} \lambda \phi \iota \tau \alpha)^{39}$. Dans cette perspective, nous pouvons à présent reprendre la petite histoire du dialogue entre Numa et Jupiter.

Numa avait réussi à «évoquer» (elicere) Jupiter. Comme nous l'avons dit, le roi voulait que le dieu lui révèle le rituel permettant d'«expier» (procurare) la foudre. Le dieu apparaît, ayant été « évoqué » (c'est pourquoi il portera le nom de Elicius), mais le dialogue entre ces deux personnages prend la forme inattendue d'un

38. vv. 18 sq.

39. Quant à Virgile, dont nous avons déjà rappelé les Bucoliques, il est intéressant de voir que sa magicienne tend à renforcer le rapport d'équivalence entre le matériel qu'elle utilise pour son rite magique et l'objet auquel est destiné l'enchantement, non tant au moyen d'une analogie phonique, mais plutôt en usant d'équivalences sémantiques : autrement dit, en utilisant le passage du grec au latin. Voici la femme abandonnée, qui, après avoir accompli divers autres enchantements pour ramener chez elle son Delphis, se produit dans ce calembour (Bucolica 8, 83): Daphnis me malus urit, ego hanc in Daphnide laurum « Le cruel Daphnis (en grec, "laurier") me fait brûler, et moi je brûle ce laurier à Daphnis (= "laurier") ». Le procédé de Virgile ne se fonde pas tant sur le signifiant (Daphnis et laurus ne se ressemblent pas phonétiquement), il repose sur le signifié : Daphnis et laurus ont le même sens dans deux langues différentes. Un exemple similaire de jeu sémantique entre le grec et le latin se vérifie aussi au vers 107 : quand l'amant finalement s'approche, l'enchanteresse commente nescio quid certe est, et Hylax in limine latrat, « quelque chose arrive, et Hylax aboie sur le seuil ». Le chien porte le nom grec - Hylax - de l'action qu'il accomplit, à savoir latrare, vi $\lambda \alpha x \tau \varepsilon \tilde{\imath}$. 
échange de calembours. Jupiter prescrit un sacrifice humain - Numa joue sur les mots, transformant chaque fois les prescriptions du dieu en pratiques bien plus inoffensives. Lisons la version de Valerius Antias $^{40}$ : «Les éclairs, tu les expieras avec une tête - d'oignon ! d'homme (...) - tu auras les cheveux ! Je voulais dire que ce soit un animal [= un être animé] (...) - alors un anchois ! » À ce point, Jupiter doit s'avouer vaincu et admet que, désormais, la foudre pourra s'expier au moyen de maena, capillo, caepicio «l'anchois, les cheveux et l'oignon ».

Nous avons donc affaire à une série de calembours tous fondés sur le même mécanisme linguistique : Jupiter émet des ordres, qui contiennent un substantif/adjectif dont le régime grammatical reste en quelque sorte ouvert. Numa en profite pour le compléter, détournant l'ordre dans une direction qui lui est plus favorable. Dans cette petite histoire, cependant, l'homophonie de type magique joue un rôle très significatif : la «tête d'homme » devient « oignon» sur la base d'une forte homophonie caput/caepicium $^{41}$; le glissement de humano en capillo ne se réalise pas seulement sur l'axe syntagmatique (Jupiter entendait humano - capite, Numa rétorque humano - capillo), mais aussi sur le plan phonique (capillo/capite) ; il en va de même pour le glissement de animalis à maena, qui ne se réalise pas seulement sur l'axe sémantique (la maena est un «être animé »), mais aussi sur l'axe phonique (a-ni-ma-lis/mae-na). La sélection des moyens rituels par lesquels purifier la foudre, en somme, passe à travers les processus de la magie homéophonique.

Pour nous rapprocher de notre texte de départ - le rituel qu'opère la vieille femme en l'honneur de Tacita dans les Fastes -, nous

40. « expiabis » dixe « capite fulgurita »; regem respondisse « caepicio »; Iovem rursus «humano»; rettulisse regem « sed capillo»; deum contra « animali »; < «maena» > subiecisse Pompilium. Cf. supra note 27. L'insertion de maena, sur la base du texte de Plutarque et surtout du maena cité de suite après dans le texte d'Arnobe, est sûre : $c f$. l'apparat critique de Concetto Marchesi, Arnobii adversus nationes libri VII, Paravia, Torino, s. d.

41. Isidore, Origines, 17, 10, 2 : cepa vocatur quia non aliud est nisi caput. 
pouvons maintenant prendre connaissance d'une conjuration rapportée dans une tabella defixionis ${ }^{42}$ :

«Muettes Silencieuses! Que Quartus reste muet, qu'affolé, il erre comme une souris en fuite ou un oiseau devant du basilic. Que sa bouche reste muette, Muettes! Que les Muettes soient funestes! Que les muettes restent silencieuses! Muettes! Que Quartus devienne fou, que Quartus soit précipité aux Erinyes et à Orcus. Que les Muettes Silencieuses restent muettes près des portes d'or. »

L'intérêt de ce texte ne réside pas seulement dans le fait qu'il contient une référence explicite à Tacita, ou mieux aux Tacitae (démontrant ainsi, si besoin est, que le rituel décrit dans les Fastes n'est pas une invention d'Ovide) : il a aussi recours à une remarquable quantité de jeux phoniques et linguistiques. Il suffit de penser à l'usage parallèle de l'adjectif mutus, répété neuf fois, en fonction de « pivot» dans diverses séquences; à sa reprise phonique à travers le participe, rare, rutus (/mutus) ; enfin à l'évocation de la « souris » (ut mus), dont la présence ne semble due qu'aux principes de la magie homéophonique (ut mutus... ut mus...ut... mutum...) $)^{43}$. Le malheureux Quartus sera rendu mutus par l'entremise de nomina aptes à susciter cette condition de prime abord au niveau phonique.

Mais revenons au processus magique décrit par Apulée dans l'Apologie : à savoir, le jeu sur la propinquitas entre les nomina en vue d'obtenir une similis vis entre les res. L'analyse des exemples donnés à ce propos par l'auteur nous montrera que les procédures de la magie homéophonique pouvaient parfois prendre la forme de

42. Chapot, Laurot, op. cit., L78 (sur une tablette de plomb trouvée à Kempten, en Bavière, en 1953) :

Mutae Tacitae!

ut mutus sit Quartus,

agitatus erret ut mus fugiens aut avis adversus basyliscum.

Ut eius os mutus sit, Mutae!

Mutae dirae sint!

Mutae tacitae sint!

Mutae! Quartus ut insaniat,

ut Eriniis rutus sit Quartus et Orco.

Ut Mutae Tacitae

ut mutae sint ad portas aureas.

Voir aussi l'analyse de Tupet, op. cit., p. 412 sq.

43. Tupet, op. cit., p. 412 sq. ; Chapot, Laurot, op. cit., ad locum. 
véritables pun, de devinettes, voire de charades. Ce qui nous rapprochera finalement de notre objectif, à savoir l'explication, par le truchement de la magie homéophonique, de quelques-unes des opérations accomplies par la vieille femme d'Ovide.

Apulée, on le sait, avait été accusé de s'être procuré deux res marinae dans le but de les utiliser comme instruments de magie. Et son choix aurait été dicté précisément par des raisons de similarité phonique (selon le principe qu'il énonçait lui-même plus haut) ${ }^{44}$ : «Vous dites qu'ont un pouvoir en amour les spuria (nom d'un mollusque et " génital féminin ») et les fascina (nom d'un mollusque et "génital masculin») à cause de la ressemblance entre leurs noms : et pourquoi de la même plage ne pourrait-on pas prélever un petit caillou pour soigner la vessie, un coquillage pour influencer un testament, un crabe pour soigner les ulcères, une algue pour soigner la fièvre qui donne des frissons ? » Il est bon de rappeler qu'Apulée n'est pas seulement sarcastique, il construit des procédés magiques tout à fait crédibles ${ }^{45}$. Toutefois, la compréhension de ces enchantements homéophoniques demande un certain effort d'imagination: le rapport entre les deux res apparaît par l'intermédiaire d'un rapport entre deux nomina dont le second n'est pas immédiatement visible, il faut en quelque sorte les « deviner ». Le calculus soigne la vessie parce que ce mot signifie «petit caillou», mais aussi « calcul vésical »; la testa (coquillage) influence le testamentum parce qu'elle constitue la première partie de ce mot (son apocope, aurait dit la terminologie antique); le cancer soigne les ulcera parce qu'il faut savoir qu'ils portent aussi le nom de carcinomata. Le cas le plus intéressant, ceci dit, est constitué précisément par l'alga. À première vue, en effet, on n'arrive pas à comprendre pourquoi une alga pourrait faire du bien à une fièvre définie comme quercerus. Passe encore s'il s'agissait du feuillage d'un quercus!

44. Apulée, Apologia, 35 : Posse dicitis ad res venerias sumpta de mari spuria et fascina propter nominum similitudinem: qui minus possit ex eodem litore calculus ad vesicam, testa ad testamentum, cancer ad ulcera, alga ad quercerum ? ; cf. discussion dans Manselli, op. cit.

45. Cf. Manselli, op. cit.; Pline soutient lui aussi que le cancer puisse soigner le « cancer », c'est-à-dire les ulcères, dans Naturalis historia, 32, 134 : cf. Abt, op. cit., p. 224 sq. 
Mais on aurait du mal à en trouver sur la plage. Pour élucider la valeur magique de l'alga, il nous faut penser que le quercerus est une fièvre algida, une fièvre qui fait frissonner de froid ${ }^{46}$. La procédure magique prend en somme la forme de la charade - le bénéficiaire de cet enchantement doit avant tout deviner un élément caché dans son énonciation. Dans des cas de ce genre, il est vraiment difficile de distinguer entre enchantement et devinette, entre charade et magie.

\section{DES FÈVES ET DES SOURIS}

Revenons donc aux Fastes d'Ovide et au rituel qu'une vieille femme adresse à Tacita pour bloquer les mauvaises langues. Nous l'avons vu, il restait à élucider deux moments que les commentateurs ne réussissent pas à expliquer sur la base des procédures magiques usuelles : l'acte de «mâchonner en bouche sept fèves » et celui de « placer trois grains d'encens sous le seuil, à l'endroit où une souris s'est ouvert un passage ». Nous avions émis l'hypothèse que, tout en restant à l'intérieur de la magie homéopathique, Ovide aurait produit ici une sorte de fracture à l'intérieur des procédures magiques : passant de la similarité entre des objets et des actions à la similarité entre des mots et des sons. Voyons donc si le texte permet d'expliquer ces mystérieuses opérations accomplies par la vieille femme, sur la base de la magie homéophonique. On peut maintenant deviner que les favae qu'elle mastique et que les trois tura (grains d'encens) qu'elle place précisément dans le passage creusé par un mus, fonctionnent à la manière du mus, dont l'évocation ne sert qu'à rendre mutus, de la testa qui influence le testa-mentum ou de l'alga qui soigne le quercerus, fièvre algida.

Nous voici donc en présence d'une vieille femme qui septem nigras versat in ore fabas "mâchonne dans sa bouche sept fèves noires ». Le rapport entre le geste magique et le but de l'enchantement - faire taire les mauvaises langues - n'est guère évident. Mais, pour en trouver de suite un, il suffit de se rappeler un des termes par

46. Cf. Corpus Glossariorum Latinorum, 4, 158, 10 : febris acris ac vehemens quae cum magno frigore solet venire; Abt, op. cit., p. 224 sq. 
lesquels on définissait à Rome le ragot : c'est-à-dire rumor. Comme je l'ai écrit ailleurs ${ }^{47}$, l'étymologie de ce terme a un rapport avec rumen, ou l'acte de ruminer, de "mâchonner », des animaux ruminants. On «mâche » et on « remâche » les mêmes aliments, voire les mêmes discours. Si la vieille versat in ore des fèves, nous la surprenons donc précisément dans l'action de «ruminer», de "mâchonner». En d'autres termes, "mâchant et remâchant en bouche ", la vieille imite l'acte de ruminer dans le sens de «qui produit des rumores », des ragots et des médisances. Le jeu est renforcé par l'existence de l'expression latine in ore esse ou in ore haberi, signifiant, à propos de quelqu'un, «être dans la bouche des gens », dans le sens où cette personne fait l'objet de rumores et de bavardages malveillants ${ }^{48}$. En ruminant, la vieille mime à la fois le geste de ceux qui produisent des rumores et l'action d' " avoir en bouche », de «parler de ». Elle représente magiquement les bouches qui ruminent leurs potins malveillants, ceux que le rituel en l'honneur de Tacita entend bloquer.

Il nous vient à ce point une autre curiosité : pourquoi la vieille rumine-t-elle précisément septem nigrae... fabae, « sept fèves noires »? Si le fait que ces fèves soient au nombre de sept n'est guère surprenant - sept est un nombre magique ${ }^{49}-$, il n'en va pas de même pour le type de nourriture choisi pour cette « rumination » magique. Le texte cache probablement un autre jeu de mots. Le latin possédait, en effet, un autre terme intéressant pour désigner les bavardages ou les ragots : fabula. Du point de vue étymologique, on ne peut établir aucun rapport entre $f a b a$ « fève » (le premier - $a$ est bref) et fabula « potin » (le premier - $a$ - est long) ; mais Ovide a pu s'amuser à en établir un. D'ailleurs, le diminutif fabula (ou fabulus) «petite fève », homophone de fabula « ragot », existe

47. Maurizio Bettini, Parole potenti, parole screditate. L'atto del fari nella cultura romana, in La potenza della parola. Destinatari, funzioni, bersagli, a cura di Simone Beta, I quaderni del ramo d'oro, 6, Cadmo, Firenze, 2004, p. 33-78.

48. Cf. Thesaurus Linguae Latinae, IX-2, 1081-1082 (Tessner). Voir l'expression française équivalente : «être sur toutes les bouches » (mais le processus ici est différent).

49. $C f$. les observations de Bömer, op. cit., II, 126. 
réellement en latin ${ }^{50}$. Dans son Poenulus, Plaute s'amuse à jouer sur le double sens du mot ${ }^{51}$ : « Vous qui avez mangé, vous avez beaucoup mieux fait ; vous qui n'avez pas mangé, vous vous rassasierez des fables comiques (/fèves). » Ensuite : «Maintenant il faut l'assaisonnement définitif de toute fable comique (/fève) ; si la pièce vous a plu, elle réclame vos applaudissements. » De son côté, Brutus écrivait à Cicéron qu' « il voulait qu'il [Cicéron] goûte (degustasses) quelque chose de ces ragots (fabulae) ${ }^{52}$ ». Quant à la couleur «noire » de ces fèves/ragots, elle pourrait faire allusion au caractère négatif - « hostile », « ennemi », comme le définit la protagoniste des bavardages qu' on entend bloquer ${ }^{53}$. L'image de la vieille qui, en mâchonnant en bouche sept fèves noires, en réalité, "grommelle des fabulae hostiles ", "mâchonne de malveillants rumores », est plutôt amusante. Au moyen de cet autre calembour de type magique, l'opération accomplie par la vieille devient un véritable concentré d'imitation/évocation de la médisance.

Considérons une autre séquence de ce rituel qui se prête à une analyse fondée sur la magie verbale : les «trois grains d'encens » placés « avec trois doigts sous le seuil » à l'endroit où une « souris s'est creusé un passage ». Commençons par les trois grains d'encens (tria tura). Qu'ils soient au nombre de « trois », et qu'on les place «avec trois doigts » est aussi peu surprenant que la présence de sept fèves « ruminées ». Comme le chiffre sept, le chiffre trois est magique ${ }^{54}$. Mieux vaut traiter l'encens, en latin tus turis, et son pluriel que l'on trouve dans le texte, tura $^{55}$. Frazer en motivait la

50. Stichus, 690.

51. Plaute, Poenulus, 7 sq. : qui edisti multo fecistis sapientius/qui non edistis, saturi fite fabulis ; $1370 \mathrm{sq}$. : num quod postremo est condimentum fabulae/si placuit, plausum postulat comoedia ; cf. aussi Stichus, 689 sq.

52. Cicéron, Ad Atticum, 13, 40, 1 : ac vellem aliquid degustasses de fabulis (les mots de Brutus sont rapportés par Cicéron); Apulée, Métamorphoses, I, 26 : evasi aliquando rancidi senis non cibo gravatus, cenatus solis fabulis.

53. Rappelons l'usage de niger dans le sens de «mauvais », « malveillant» dans des contextes comme Horace, Saturae, 1, 4, 81 ; Sénèque, Phèdre, 493 (niger livor « noire envie »).

54. Cf. Bömer, op. cit., II, 126.

55. La magicienne de Virgile aussi brûle de l'encens avant le début de la cérémonie, $c f$. Bucolica, 8, 65. 
présence par le culte des morts, mais qui sait si cet encens n'agissait pas plutôt en l'honneur du démon de l'analogie ? En latin, le verbe «boucher» se dit ob-turo (sans rapport avec la racine de tus turis, bien sûr, sur le plan étymologique), ce qui permet d'expliquer pourquoi la vieille dépose trois tura à l'endroit où une souris s'est ouvert un passage : au moyen des tura, elle $o b$-turat « obture » le passage, le «ferme », comme elle l'a fait en utilisant de la poix et en cousant la tête de maena.

Admettons qu'il en soit ainsi pour les tria tura ${ }^{56}$. Mais que dire de la souris, en latin mus muris? Si l'on y pense bien, cette souris est vraiment le personnage le plus énigmatique de cette affaire.

Pour fournir une explication à cette curieuse présence, nous pourrions avoir recours au terme mus que la magie homéophonique a déjà placé sur notre route dans la tabella defixionis : un mus se trouverait comme un charme dans un rituel en l'honneur d'une déesse muta, muette, et surtout dans l'intention de rendre « muettes » (mutae) les bouches médisantes. Pourtant, c'est un autre terme encore qui nous vient à l'esprit : pour définir le ragot, outre rumor et fabula, les Latins possédaient le mot murmur, d'où murmurare et murmuratio. Le signifiant de murmur se présente comme un redoublement parfait du thème mur - de mus muris « souris »-sans qu'il existe entre ces deux termes de réelle correspondance étymologique. Il n'empêche que, du point de vue purement phonétique, le ragot, ou murmure, se présente en latin comme une « souris-souris ». Donc, lorsqu'elle place des grains d' «encens » (tura) dans le trou creusé par une «souris » (mus muris), l'exécutante ne fait qu' «obturer, boucher, le murmure », obturer le canal qui, en tant que creusé par un mus, représente le murmur. En somme, la vieille bloque les ragots « en encensant une souris-souris »-quoi de plus intraduisible qu'un calembour! Mais une autre rencontre sonore peut encore renforcer ce jeu de mots : dans la langue latine le terme mur-mur se croise phonétiquement avec un autre mot signifiant le ragot, à savoir ru-musculus, diminutif de rumor. En restant sur le plan de la phonétique, il est facile de reconnaître dans le terme ru-musculus,

56. Observons dans ce vers (573), l'augmentation de la densité des figures sonores : digi-tis tria tura tribus. 
le diminutif de mus, à savoir musculus « petite souris ». Ovide spécifie clairement que le mus en question est brevis, «petit»-en somme, c'est un musculus.

Voilà donc une façon bien curieuse, et bien amusante, de se mettre à l'abri des ragots, des bavardages et des médisances. Au moyen de calembours et de jeux de mots qui «miment» phonétiquement, d'une part, la production de ces bavardages et ragots, et, d'autre part, l'action contraire de les bloquer, la vieille entend mettre fin aux médisances en honorant une déesse qui «fait taire». Selon le principe énoncé par Apulée, cette magicienne cherche la propinquitas nominum pour déchaîner une vis similis qui agisse sur la res. En général, on pourrait naturellement observer que cette façon de procéder est d'un style très ovidien, digne du poète qui, surtout dans les Fastes ${ }^{57}$, n'a pas peur de jouer avec les mots : autrement dit, pour le dire à la manière de la critique anglo-saxonne, Ovide pratique volontiers le pun. Du reste, il le fait dans la suite du texte, en produisant une série de jeux de mots fondés sur $\lambda \alpha \lambda \varepsilon \tilde{\imath} \nu$, Lala, Lara et Lares. Mais, dans le cas du rituel adressé à Tacita, il est nécessaire de souligner la spécificité du contexte dans lequel ces calembours sont produits.

Nous n'avons pas affaire à la narration d'un mythe, ni à une déclaration de poétique, ni à une louange adressée à Auguste : nous assistons à la mise en scène d'un rituel magique. Nous sommes en présence d'un de ces moments où la littérature s'empare de la magie, pour en faire son objet; et la magie s'insinue dans les plis du texte littéraire, pour lui attribuer ses cadences uniques. Dans des cas comme le rituel ovidien en l'honneur de Tacita, les jeux phoniques - « ruminer des fèves/de perfides ragots », «obturer/encenser les murmures/ souris »- ne doivent pas être pris pour un simple ludus littéraire, mais comme une façon de transférer les procédés analogiques typiques de la magie au niveau du langage de la littérature. En hommage à la terminologie critique, qui aime toujours la précision, nous pourrions dire qu'il s'agit de pun à caractère de charm; autrement dit, des pun

57. Je me réfère en particulier à l'essai de Alessandro Barchiesi, Il poeta e il principe, Laterza, Bari, 1994. 
qui, dans leur procédure analogique, ont une intention incantatoire. Des jeux de mots où la pragmatique linguistique va de pair avec l'allusion ludique ${ }^{58}$.

Centro Antropologia e Mondo Antico

Facoltà di Lettere

Via Roma 47

53100 Siena

58. Ce texte a été discuté à Paris dans le cadre du Groupe de Recherches « Antiquité au Présent » de l'Équipe « Théorie Littéraire et Sciences humaines » dirigée par Florence Dupont. Je remercie Annie Dubourdieu, Maxime Pierre, Florence Dupont et tous les participants pour les suggestions qu'ils ont bien voulu me faire. 